\title{
Near-field scanning optical microscopy using polymethylmethacrylate optical fiber probes
}

\author{
H. Chibani, K. Dukenbayev, M. Mensi, S.K. Sekatskii *, G. Dietler \\ Laboratoire de Physique de la Matière Vivante, IPSB, BSP, Ecole Polytechnique Fédérale de Lausanne, CH1015 Lausanne, Switzerland
}

\section{A R T I C L E I N F O}

\section{Article history:}

Received 23 July 2009

Received in revised form

4 November 2009

Accepted 18 November 2009

Keywords:

Scanning near-field optical microscopy

Plastic optical fibers

\begin{abstract}
A B S T R A C T
We report the first use of polymethylmethacrylate (PMMA) optical fiber-made probes for scanning near-field optical microscopy (SNOM). The sharp tips were prepared by chemical etching of the fibers in ethyl acetate, and the probes were prepared by proper gluing of sharpened fibers onto the tuning fork in the conditions of the double resonance (working frequency of a tuning fork coincides with the resonance frequency of dithering of the free-standing part of the fiber) reported earlier for the case of glass fibers. Quality factors of the probes in the range 2000-6000 were obtained, which enables the realization of an excellent topographical resolution including state-of-art imaging of single DNA molecules. Near-field optical performance of the microscope is illustrated by the Photon Scanning Tunneling Microscope images of fluorescent beads with a diameter of $100 \mathrm{~nm}$. The preparation of these plastic fiber probes proved to be easy, needs no hazardous material and/or procedures, and typical lifetime of a probe essentially exceeds that characteristic for the glass fiber probe.
\end{abstract}

(c) 2009 Elsevier B.V. All rights reserved.

\section{Introduction}

Scanning near-field optical microscopy (SNOM) is capable of measuring optical properties on a subwavelength scale exceeding the Abbe diffraction limit with simultaneous topography imaging with a nanometer spatial resolution. Nowadays it is widely used to study a broad variety of samples in biology, material science, nanotechnology and other fields; see e.g. [1-4] for recent reviews.

By far the most popular SNOM probe is a metal-coated tapered optical glass fiber on which apex an aperture for the light transmission, whose diameter is between 50 and $200 \mathrm{~nm}$, is formed. The optical resolution is of the order of the aperture size. Unfortunately, these probes are expensive, very fragile (not surprising for a glass-made construction) and their fabrication is difficult, hard to control and in many cases a hazardous process. Of course, the wide use of scanning near-field optical microscopy is hampered by the difficulty in producing probes with sufficient accuracy and a good reproducibility.

In this article we present first SNOM probes made from polymethylmetacrylate (PMMA) optical fibers, which nowadays rapidly gain popularity as "last one hundred meters fibers" due to their cheapness and easiness to work with, see e.g. $[5,6]$. Concerning the SNOM domain, these fibers look very promising for use as SNOM probes as one can anticipate much less fragility

\footnotetext{
* Corresponding author. Tel.: +4121693 0445; fax: +41216930422.

E-mail address: serguei.sekatski@epfl.ch (S.K. Sekatskii).
}

(compare glass and plastic) and greater ease of tip preparation. Actually, for such a preparation, hazardous HF treating, which remains the most popular approach to prepare SNOM probes from the glass fibers [1-4], can be completely avoided. These predictions have been confirmed by experiments and the results of these are presented below.

\section{Experimental}

The polymethylmethacrylate optical fibers (POF) used in this study were purchased from A.R.T. Photonics $\mathrm{GmbH}$, Germany. They are step index-type fibers with a diameter of $250 \mu \mathrm{m}$ made of high-purity PMMA core, with an index of refraction of $n_{\text {core }}=1.49$, and a fluorinated PMMA cladding with an index of refraction of $n_{\text {cladding }}=1.35$.

A 99.5\% ethyl acetate (EA, Aldrich) was chosen as an etchant because weight loss of POF in it was observed to be the most significant and because the dissolution rate of POF in EA is almost linear with immersion time. In this sense our results coincide with the data presented in [7]. A two-stages etching procedure was used. At the first stage the ca. $2 \mathrm{~cm}$-length portion of the fiber was submerged into ethyl acetate for $30-40 \mathrm{~min}$. As a result, the diameter of the fiber diminished ca. twice and the external protective polymer film coat of the fiber was destroyed. At the second stage (20-30 min) the end part of the bare fiber has been slowly submerged into the etching solution with the periodical agitation of the solvent-containing glass. Such a procedure results 
in the formation of the sharp conical tip with the radius of curvature equal to $50-100 \mathrm{~nm}$ and aspect ratio 8-10 [8]. After etching, the tips were rinsed in de-ionised water.

A scanning electron microscope image of a typical etched POF probe coated with a gold nanolayer to avoid charge effects during the imaging is presented in Fig. 1.

Sharpened POF probes were glued onto the tuning fork using the "double resonance" principle of operation first introduced in [9] and then successfully used for Scanning Near-field Optical Microscopy of different samples [10,11]. This mode of operation relies specifically on the arrangement of the fiber probe onto the tuning fork (see inset in Fig. 2) so that the working frequency of the latter coincides with the resonant frequency of lateral dithering of the free-standing part of the fiber beam $b$ properly glued onto the tuning fork metal case $a$. In the paper [9] we experimentally demonstrated that such an assembling enables to preserve a large quality factor of the tuning fork after gluing the glass fiber probe onto it and also presented a theoretical model explaining such preservation.

Double resonant operation requires accurate gluing and careful control of the length of the free-standing part of the fiber beam as well as the position where the thin glass connector between the probe and the tuning fork is to be glued. It was experimentally established that for a standard $125 \mu \mathrm{m}$-diameter sharpened glass fiber, the resonance condition holds when the length of a freestanding part of the fiber beam is equal to $4.8 \pm 0.1 \mathrm{~mm}$ [9]. Based on the well-known formula expressing the resonant frequencies of the dithering of a cylindrical beam whose one end is clamped and other is free, $\omega=\left(\alpha_{n}^{2} / l^{2}\right) \sqrt{E I / \rho S}$ (here $I=(1 / 4)\left(\pi r^{4}\right)$ is the moment of inertia, $l$ and $r$ are the length and the radius of the cylindrical rod, respectively, $E$ and $\rho$ are, respectively, Young's modulus and density of the material it is made of, $S=\pi r^{2}$ and $\alpha_{n}$ is a numerical constant characterizing $n$th resonance: $\alpha_{1}=1.875$, $\alpha_{2}=4.694$, etc. [12]), we obtain the scaling rule how this double resonant arrangement is to be effected for different materials: for this the number $\eta=\left(r / l^{2}\right) \sqrt{E / \rho}$ should be kept constant. This formula was the starting point to determine the optimal probe parameters, but it turned out that both Young's modulus and density of PMMA varied broadly. Hence careful experimental adjustment of the plastic fiber probe emplacement was necessary. The best results were obtained when the diameter of a probe was around $0.125 \mathrm{~mm}$ (twice diminished by etching in comparison with the initial diameter, see above) and the length was equal to $3.4 \pm 0.1 \mathrm{~mm}$. The distance between the drive $d$ (Fig. 2; glass rod a

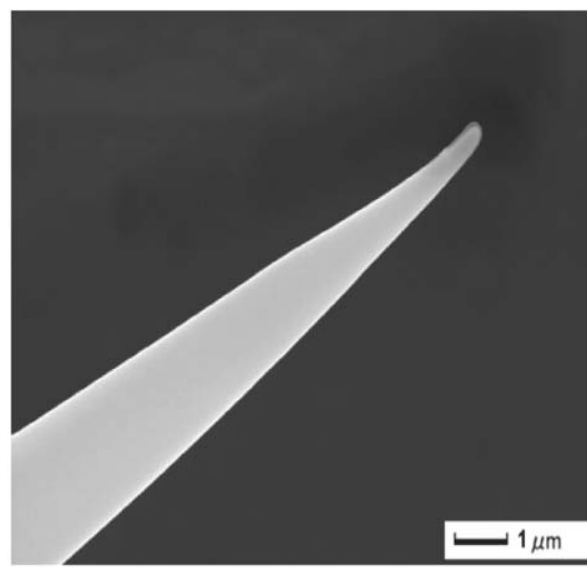

b

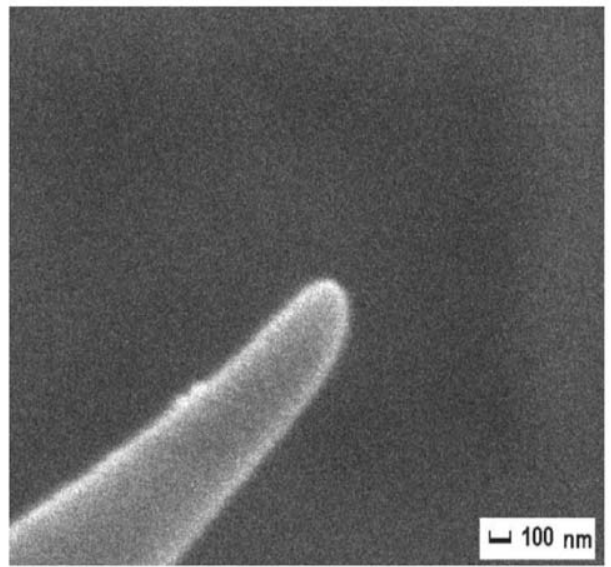

Fig.1. Scanning electron microscopy images of an etched POF nanoprobe.

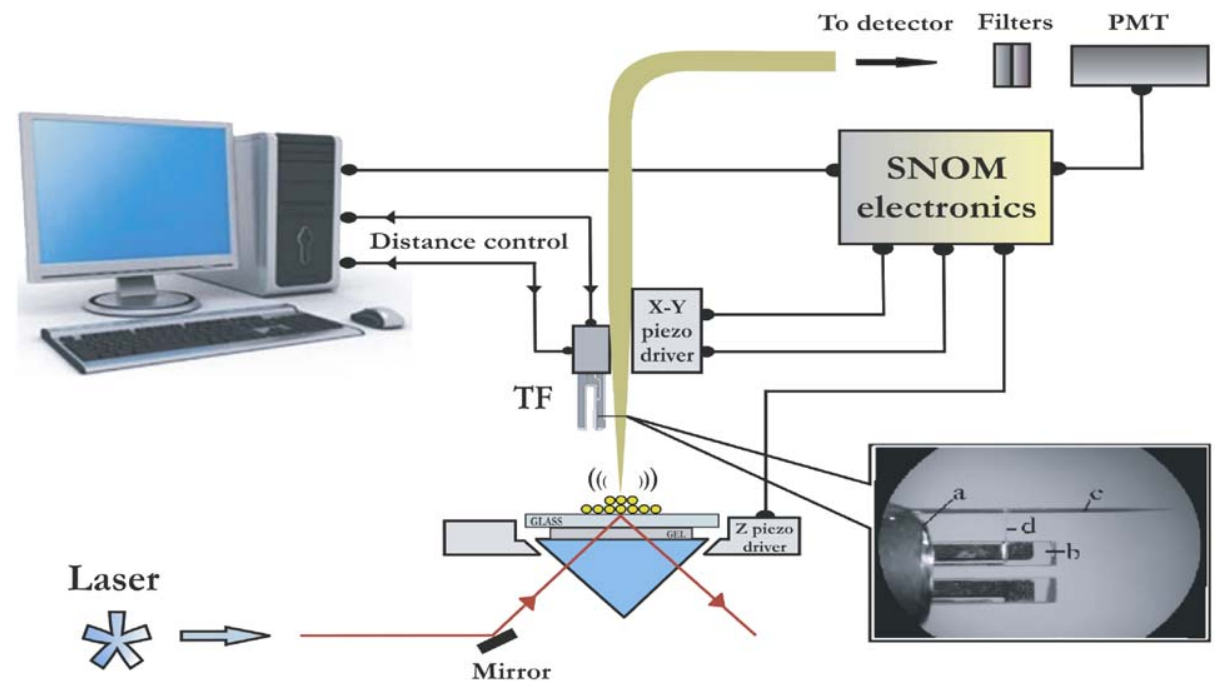

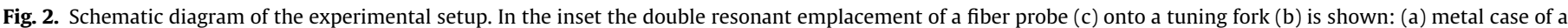
tuning fork and (d) thin glass rod connecting probe and tuning fork. 
with the diameter of $20 \mu \mathrm{m}$ was used as a drive) and the tuning fork case was fixed at $1.4 \pm 0.1 \mathrm{~mm}$.

The result of accurate probe/tuning fork coupling is a large quality factor, $Q$ of the sensor: the initial values of $Q$ in 10,00011,000 , which is characteristic for a free unloaded tuning fork in air, drops down to the values $Q$ in 2000-6000 after proper gluing of a glass fiber probe onto it. These values are quite comparable with those obtained earlier for glass fibers [9]. Attainment of such large quality factors enables us to use the specialized low noise, precise and fast electronics to measure the resonant frequency $f_{\text {res }}$ and $Q$-factor of a tuning fork to control the SNOM operation. Both the proprietary electronics earlier designed essentially for the sensors made by a tuning fork and an AFM cantilever glued on it $[10,13]$, and a commercially available electronics equipment from Nanonis SA, Switzerland, specially adapted to work with this particular SNOM, were exploited.

To avoid complications related with the necessity to prepare metal-coated sharpened aperture probe, at this demonstration stage, we used a Photon Scanning Tunneling Microscope (PSTM) $[1-4,14]$ for the operation in the near field, see Fig. 2. In PSTM, the sample is illuminated by an evanescent wave produced by total internal reflection, and the optical field of the sample is probed by locally frustrating the evanescent field with an uncoated tapered optical fiber probe. Essentially, the same SNOM as reported by us in [10] was adapted to operate in this PSTM regime. We do not specially study the efficiency of the light collection by our plastic probes but qualitatively it does not differ much from that of standard fiber probes exploited in the same conditions [14]. The samples were deposited onto thin glass plate placed onto the glass prism surface with a drop of the index matching gel (Cargille Laboratories, Ceder Grove, NJ, USA). A $488 \mathrm{~nm}$ argon ion laser whose power is diminished to $2.5 \mathrm{~mW}$ (Spectra Physics) was used to illuminate the sample. A $488 \mathrm{~nm}$ Holographic Notch filter (Kaiser Optical Systems, Inc., USA) was inserted to cut off the excitation laser light. Fluorescence was detected by the PMT MP942 (Perkin Elmer) working in single photon counting mode.

\section{Results and discussion}

In Fig. 3 we present shear force topographical images of a TGQ 1 calibration grating (NT-MDT Company, Russia) and densely packed $1.0 \mu \mathrm{m}$ diameter fluorescent polystyrene microspheres $488 / 560 \mathrm{~nm}$ (T-8880, Molecular Probes Inc., Oregon, USA) on a thin glass slide surface obtained using PMMA fiber probes and the SNOM under discussion. To better illustrate the performance of our SNOM, in Fig. 4, we present a shear force topographical image of single Lambda Mix Marker 19 \#SM0231 DNA molecules (Fermentas, Ontario, Canada), which in our opinion is among the best topographical images of DNA molecules obtained by a scanning probe microscopy of any type. DNA molecules with the concentration of $1 \mu \mathrm{g} / \mathrm{ml}$ were deposited onto the APTESfunctionalized mica surface as discussed in [15]. High topographical resolution of these images demonstrates that a few nanometer-size "whiskers" and/or other irregularities are often present on the surface of generally smooth (see Fig. 1) PMMA tip apex.

Densely packed fluorescent beads in Fig. 3 were prepared by simple drying of a drop of a bead solution on the glass slide surface. This results in multilayer bead coating which prevents the attainment of the near-field optical resolution. Such resolution is illustrated in Fig. 5 where the isolated fluorescent

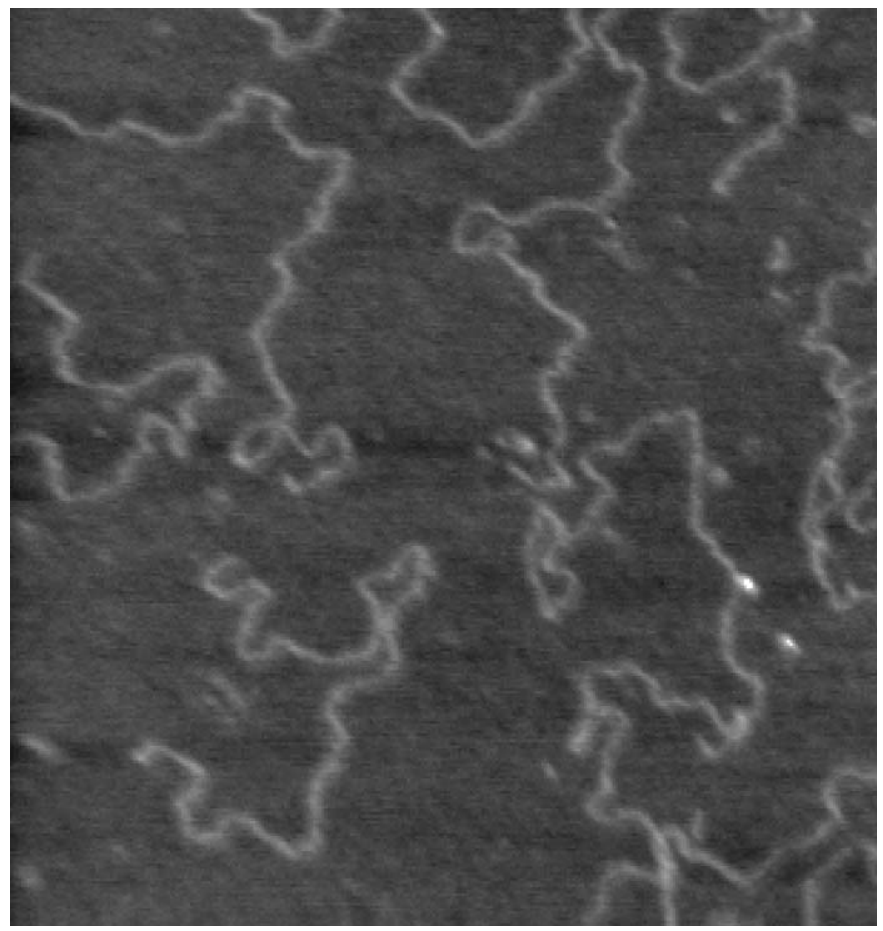

Fig. 4. Shear force topographical image of single Lambda DNA molecules on mica. Constant amplitude mode of the Nanonis SA, Switzerland. SNOM controller was used as a feedback source. Scan size: $2.2 \times 2.2 \mu \mathrm{m}$.
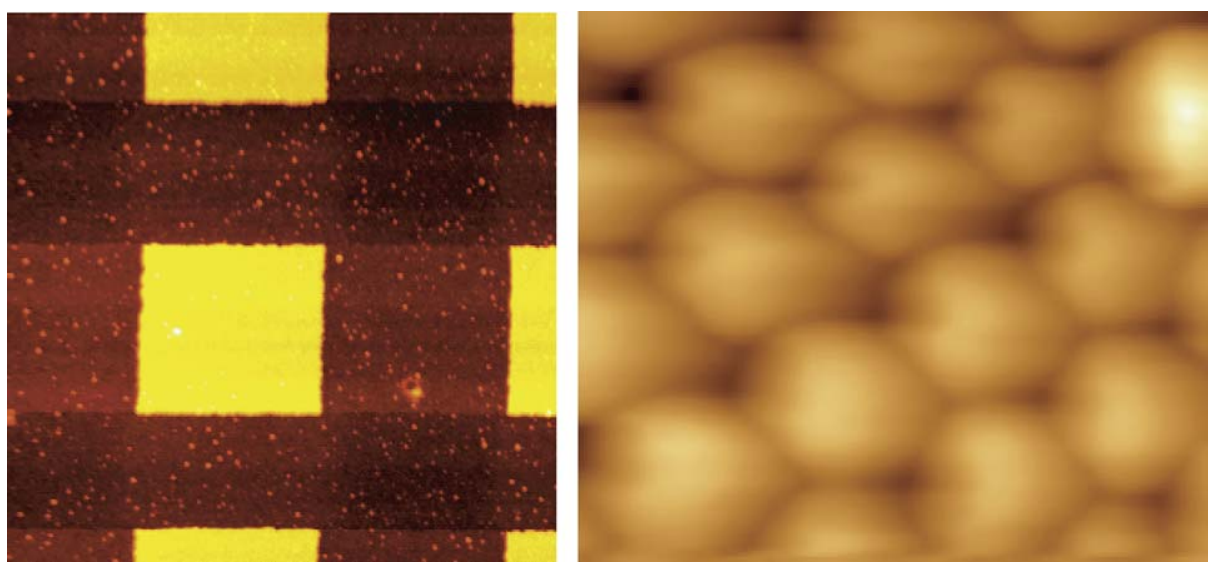

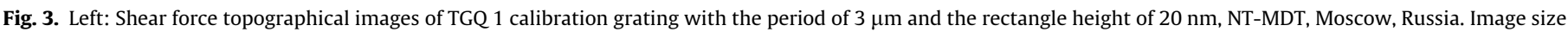

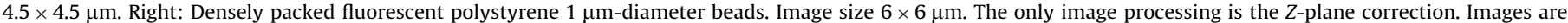
presented without the scanner nonlinearity compensation. 
a

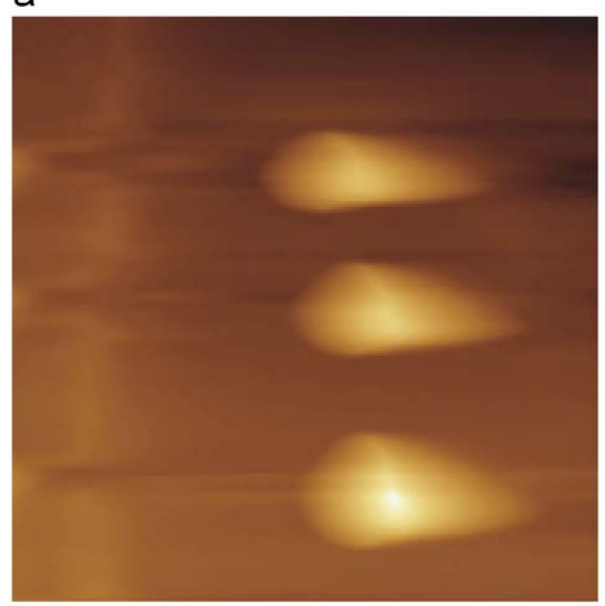

C

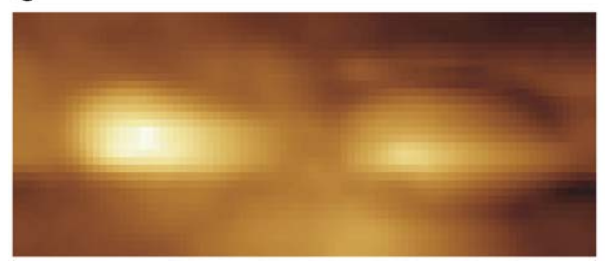

b

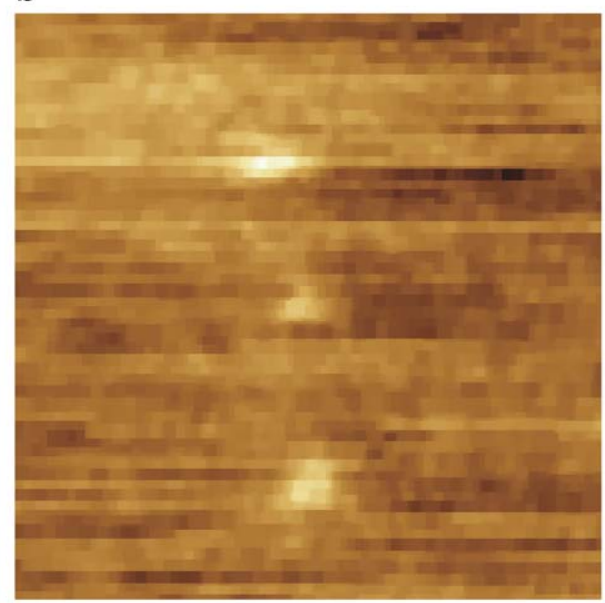

d

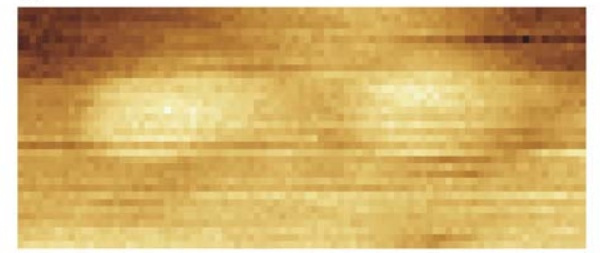

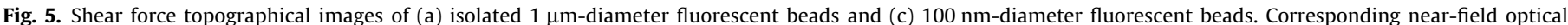

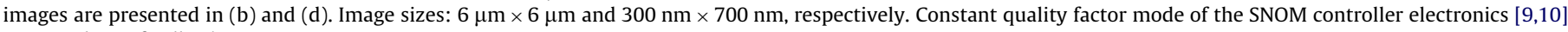
was used as a feedback source.

beads are seen. These samples were prepared as follows. Water solutions of fluorescent polystyrene microspheres $488 / 560 \mathrm{~nm}$ of $0.1 \mu \mathrm{m}$ and $1 \mu \mathrm{m}$ diameters (T-8872 and T-8880, respectively, Molecular Probes Inc., Oregon, USA) with the initial concentration $2 \%$ vol. were, each, diluted 1000 times. Then one drop (10-20 $\mu \mathrm{l})$ of each solution was deposited on the glass slide surface and dried in air. Beforehand these glass slides were pretreated with $0.1 \%$ poly-L-lysine solution (P8920, Sigma-Aldrich) by submerging them into this solution for $30 \mathrm{~min}$ and then drying in air for 5-6 h. As follows from experimental results, such a deposition procedure enables the preparation of the samples where isolated fluorescent beads are present. No displacement of the beads during scanning was observed, which is due to both the reasonably strong adhesion of the beads to the poly-lysinetreated glass surface and small forces characteristic for this SNOM, see [10].

Of course, the samples under study are not flat. Hence topographical artifacts cannot be excluded, see e.g. [16,17], and these images should not be taken for the quantitative estimation of the near-field optical resolution achieved. Such quantitative study will be performed after the preparation of plastic fiber probes with the subwavelength-size aperture for light transmission (actually the first aluminum-coated PMMA aperture probes have been successfully prepared and their testing is in progress). What is important is that they, together with the topographical images presented above, unambiguously attest the successful realization of PMMA fiber probes for SNOM.

\section{Conclusion}

We have reported the first use of a PMMA fiber probes for Scanning Near-field Optical Microscopy. Preparation of these probes is easy and does not require any hazardous materials and/or procedures. The hydrophobic nature of the plastic fiber surface and relative easiness of its chemical modification also can be advantageous for certain cases. These points are well illustrated by our first results obtained when using these plastic probes in water: their use turned out to be easier than that of the glass fiber probes and these probes proved to be sufficiently chemically inert to enable a few days of exploitation without any noticeable degradation (cf. [18]; detailed report will be published elsewhere).

What is probably most important is that these probes do demonstrate much less fragility than the glass fiber-made probes. Our statistics is not yet rich enough to give exact numerical data (around 30 PMMA probes have been intensively exploited in real experimental conditions), but for one both the days it works were many times more and its mean lifetime can be estimated as 3-4 days, which, according to our experience, is at least two times better than that for similar glass fiber probes. We believe that all these factors promise broad perspectives for the probes under discussion.

\section{Acknowledgements}

The authors thank Dr. Jean-Daniel Ganière for his help with the SEM images and Dr. Josef Adamcik for the preparation of the $\lambda$-DNA sample. This study was partly supported by the Swiss National Science Foundation grant no 200020-117962/1.

\section{References}

[1] M.A. Paesler, P.J. Moyer Near Field Optics: Theory Instrumentations, and Applications, Wiley, New York, 1996.

[2] D. Courjon, in: Near-Field Microscopy and Near-Field Optics, Imperial College Press, London, 2003.

[3] H. Taha, A. Strinkovski, A. Manevitch, A. Khatchatouriants, R. Dekhter, E. Ammann, A. Lewis, Nature Biotechnology 21 (2003) 1378.

[4] A.V. Zayats, D. Richards (Eds.), Nano-optics and Near-field Optical Microscopy, Artech House, 2008. 
[5] O. Ziemann, J. Krauser, P.E. Zamzow, W. Daum, in: Plastic Optical Fiber Handbook-Optical Short Range Transmission Systems, Springer, Berlin, 2008.

[6] P. Polishuk, Fiber and Integrated Optics 27 (2008) 1.

[7] M.H. Im, E.J. Park, C.H. Kim, M.S. Lee, Human-Computer Interaction Interaction Platforms and Techniques, 4551, Springer, Berlin, Heidelberg, 2007.

[8] Dependence of the aspect ratio of the probe on the etching parameters has not yet been studied in details but preliminary data shows that it can be varied by varying the submersion rate.

[9] A.P. Cherkun, D.V. Serebryakov, S.K. Sekatskii, I.V. Morozov, V.S. Letokhov, Rev. Sci. Instrum. 77 (2006) 033703.

[10] D.V. Serebryakov, S.K. Sekatskii, A.P. Cherkun, K. Dukenbayev, I.V. Morozov, V.S. Letokhov, G. Dietler, J. Microsc. 229 (2008) 287.
[11] S.K. Sekatskii, G. Dietler, V.S. Letokhov, Chem. Phys. Lett. 452 (2008) 220

[12] L. Landau, E. Lifshitz, Theory of Elasticity, Butterworth-Heinemann, 1986.

[13] D.V. Serebryakov, A.P. Cherkun, B.A. Loginov, V.S. Letokhov, Rev. Sci. Instrum. 73 (2002) 1795.

[14] S.K. Sekatskii, G.T. Shubeita, G. Dietler, Appl. Phys. Lett. 77 (2000) 2089.

[15] J. Adamcik, F. Valle, G. Witz, K. Rechendorff, G. Dietler, Nanotechnology 19 (2008) 384016.

[16] B. Hecht, H. Bielefeldt, Y. Inouye, D.W. Pohl, L. Novotny, J. Appl. Phys. 81 (1997) 2492

[17] V. Sandoghdar, S. Wegscheider, G.G. Krausch, J. Mlynek, J. Appl. Phys. 81 (1997) 2499

[18] K. Dukenbayev, S.K. Sekatskii, D.V. Serebryakov, A.V. Zayats, G. Dietler, 2007, SPIE Proceedings, vol. 6728, ICONO 2007: Novel Photonics Materials; Optics and Optical Diagnostics of Nanostructures, SPIE Paper Number: 6728-2D. 